\title{
Razones técnicas y económicas para la implantación de la Cirugía Robótica en un Sistema Público de Salud (modelo español)
}

\author{
Martínez-Salamanca JI*, A. Allona Almagro A**. \\ *Department of Urology. Weill Medical College at Cornell University. New York, USA. \\ **Servicio de Urología. Hospital Universitario Ramón y Cajal y Hospital Ruber Internacional. Madrid.
}

Actas Urol Esp. 2007;31(6):603-610

\section{RESUMEN}

\section{RAZONES TÉCNICAS Y ECONÓMICAS PARA LA IMPLANTACIÓN DE LA CIRUGÍA ROBÓTICA EN UN SISTEMA PÚBLICO DE SALUD (MODELO ESPAÑOL)}

La prostatectomía radical (PR) en cualquiera de sus cuatro abordajes (perineal (PRP), retropúbico (PRA), laparoscópico (PRL) o robótico (PRR)) es hoy en día, el estándar de tratamiento quirúrgico del cáncer de próstata en estadios localizados. Parece claro, que los abordajes mínimamente invasivos (laparoscopia y robótica) son capaces de disminuir la estancia hospitalaria así como la pérdidas sanguíneas y por tanto la necesidad de transfusión. Asimismo, los resultados a medio plazo de la laparoscopia y a corto plazo de la robótica, parecen indicar, que son igual de seguras oncológicamente y capaces de conseguir los mismos o incluso superiores, resultados funcionales.

Nuestro objetivo ha sido evaluar el impacto económico que tienen estas técnicas para considerar su definitiva implantación, así como las ventajas e inconvenientes de su potencial implantación en nuestro Sistema Sanitario.

Palabras clave: Prostatectomía radical. Robótica. Laparóscopica. Abierta. Análisis de costes. Economía.

\section{ABSTRACT}

TECHNICAL AND ECONOMIC REASONS TO SET UP ROBOTIC SURGERY IN A PUBLIC HEALTH SYSTEM (SPANISH MODEL)

Radical prostatectomy (RP) is today, in any of its four approaches (perineal (PRP), retropubic (ARP), laparoscopic (LRP) or robotic (RRP), the standard surgical treatment in localized prostate cancer. It looks clear that the minimum invasive approaches (laparoscopy and robotics) are able to reduce hospital stay as well as blood loss and therefore transfusion requirement. Also, laparoscopic results at mid and robotics at short term, seem to indicate, that both are safe oncologically and able to obtain same or even superior, functional results. Our objective has been to evaluate the economic impact that these techniques have to consider their definitive implantation, as well as the advantages and disadvantages of its potential implementation in our Health System.

Keyword: Radical prostatectomy. Robotic. Laparoscopic. Open prostatectomy. Analysis of costs. Economics.

$\mathrm{L}$ os profesionales de la salud nos encontramos cada vez más frecuentemente, ante el gran reto de enjuiciar objetivamente las nuevas terapias y tecnologias que desembarcan, provenientes de compañías privadas, en el sector sanitario.
En muchas ocasiones, no es tarea fácil, evaluar la eficacia, eficiencia y efectividad de las mismas, sin dejarse llevar por el entusiasmo de la novedad, inquietud ésta que, por otra parte, ha sido el motor de los avances de la ciencia. 
En los inicios de este nuevo siglo, apareció en el armamentarium quirúrgico un nuevo invitado, el robot.

Han sido varios los caminos que han conducido a la cirugía robótica tal y como hoy la conocemos. El antecedente de esta tecnología más conocido por la comunidad urológica se basa en el paradigma del sistema maestro-esclavo, en el cual el cirujano realiza los movimientos en un dispositivo (consola) y el instrumento robotizado lo reproduce sobre el paciente. Ejemplos de esta tecnología eran el da Vinci Surgical System (Intuitive Surgical, Sunnyvale, California) y el Zeus Robotic Surgical System (Computer Motion, Goleta, California).

En Octubre de 2001 la FDA Norteamericana aprobó el uso del ZEUS Surgical System. Partiendo de la aplicación de su propia tecnología original, Computer Motion añadió dos tele-manipuladores robóticos controlados por el cirujano para completar el sistema. El coste de los sistemas existentes por aquel entonces AESOP, ZEUS y da Vinci, era, respectivamente de alrededor de 80.0000, 975.000 y 1.000 .0000 de dólares ${ }^{1}$. Finalmente, en junio de 2003, las compañias Intuitive Surgical y Computer Motion se fusionaron, y combinaron sus patentes y esfuerzos comerciales para promocionar las líneas de producto de ambas, quedando el sistema da Vinci perfeccionado como el único robot quirúrgico comercializado.

La prostatectomía radical (PR) en cualquiera de sus cuatro abordajes (perineal (PRP), retropúbico (PRA), laparoscópico (PRL) o robótico (PRR)) es hoy en día, el estándar de tratamiento quirúgico del cáncer de próstata en estadios localizados. En Estados Unidos, este tratamiento supone un desembolso global anual de 1,7 billones de dólares ${ }^{2}$. Parece claro, que los abordajes mínimamente invasivos (laparoscopia y robótica) son capaces de disminuir la estancia hospitalaria así como la perdidas sanguíneas y por tanto la necesidad de transfusión ${ }^{3-6}$. Asimismo, los resultados a medio plazo de la laparoscopia y a corto plazo de la robótica, parecen indicar, que son igual de seguras oncológicamente y capaces de conseguir los mismos o incluso superiores, resultados funcionales.
Tras estas consideraciones, seria interesante saber el impacto económico que tienen estas técnicas para considerar su definitiva implantación.

En general, hay multitud de referencias, algunas en nuestro país, que demuestran que en algunos procedimientos, la laparoscopia (técnica minimamente invasiva) aventaja a la cirugia convencional en estancia hospitalaria, necesidad de medicación analgésica postoperatoria y convalecencia ${ }^{7-10}$.

Además de las posibles ventajas comentadas en relación al abordaje minimamente invasivo, la tecnología robótica presenta unas características novedosas, que hacen interesante el conocer si podrian servir para, en manos expertas, mejorar los resultados funcionales en un futuro no muy lejano.

La idea inicial para el uso del robot fue intentar ayudar al cirujano a replicar la técnica de PRL más fácilmente. En sus inicios, y también ahora, la curva de aprendizaje de la PRL es ardua y requiere de gran esfuerzo por parte de cirujano, pudiendo repercutir negativamente en los resultados funcionales y oncológicos de los primeros 50 ó 100 pacientes $^{11-14}$.

Aunque la robótica fue concebida con fines militares para realizar telecirugía (el cirujano está a distancias kilométricas del paciente) y para aplicaciones en cirugía cardíaca, la urología, y concretamente la prostatectomía radical, se alzó como la indicación estrella para el uso del robot quirúrgico.

La cirugía laparoscópica asistida por robot permite superar algunas de las limitaciones técnicas de la laparoscopia clásica manteniendo para el paciente las ventajas del abordaje minimamente invasivo. Aunque la cirugía laparoscópica ha supuesto una revolución en el desarrollo de la cirugía, presenta algunos inconvenientes importantes:

1. Las técnicas laparoscópicas que precisan de suturas intracorpóreas conllevan una importante dificultad técnica y una no despreciable curva de aprendizaje. La cirugía laparoscópica clásica se realiza con una visión bidimensional del campo quirúrgico, perdiendo el cirujano la referencia de la profundidad.

2. Los puertos de acceso limitan la capacidad de maniobra, con la consiguiente pérdida de 2 grados de libertad en comparación con la cirugía abierta. 
3. El cirujano pierde la sensación táctil, que puede ser de gran ayuda en muchas situaciones.

El Sistema Quirúrgico da Vinci permite al cirujano realizar movimientos manuales en la consola quirúrgica que son digitalizados y modificados por un sistema informático que controla los brazos del robot en tiempo real, aplicando los movimientos sobre el paciente.

Este sistema ofrece varias ventajas sobre la laparoscopia convencional:

1. La consola que utiliza el cirujano está dotada de unas características ergonómicas adecuadas para la realización de la intervención quirúrgica en condiciones óptimas. El cirujano puede efectuar movimientos manuales y naturales, en lugar de movimientos contra intuitivos que tienen lugar en las incómodas posturas que a menudo obliga a adoptar la laparoscopia convencional.

2. Mediante la digitalización de los movimientos de la mano del cirujano, el sistema robótico elimina los temblores y movimientos en escalera, de esta manera, un movimiento manual amplio efectuado en la consola quirúrgica es traducido en un movimiento mínimo y preciso en el campo quirúrgico.

3. Los brazos robóticos pueden proporcionar grados de libertad adicionales en el interior del paciente, con objeto de incrementar la agilidad y precisión. En comparación con los instrumentos laparoscópicos rígidos y fijos, los brazos quirúrgicos presentan articulaciones adicionales que reproducen con mayor fidelidad los movimientos de la muñeca humana.

4. La posibilidad de una imagen tridimensional del campo quirúrgico mejora la percepción de la profundidad del campo por parte del cirujano. A ello se añade la enorme calidad de las ópticas y la magnificación 10x que ofrece.

La cirugía robótica, no sólo se está implantado de manera decidida para realizar prostatectomías radicales, sino que podría ser útil en la mayoría de los procedimientos urológicos actuales.

\footnotetext{
- Cirugía Renal

- Nefrectomía Radical y Parcial

- Pieloplastia

- Nefropexia

- Pielolitotomías
}

- Cistectomía Radical y derivación urinaria.

- Cistectomía parcial

- Colposacropexia (Prolapso genital)

- Linfadenectomía retroperitoneal

- Ureterolitectomía

- Reimplantación urétero-vesical

- Adrenalectomía

Además de la Urología, son varias las especialidades que han incorporado entre sus técnicas la cirugía robótica. De manera mas decidida, lo han hecho la Cirugía General, la Ginecología, la Cirugía Cardiaca, la Cirugía Torácica así como la Cirugía Pediátrica.

Tras esta breve introducción, el objetivo de este capítulo, es intentar dar una visión al lector, de los costes de esta tecnología así como de las posibles ventajas o inconvenientes de su introducción en nuestro Sistema Público de Salud.

\section{LIMITACIONES DE LA REFLEXIÓN}

Las limitaciones para realizar un análisis de costes de una tecnología que lleva implantada en España, poco más de un año (julio 2005) son muchas y variadas. Entre otras destacan:

- En el momento actual, existen en nuestro país, cuatro unidades da Vinci funcionando, y tan sólo una de ellas en el Sector Público.

- El desarrollo mayor de estos sistemas se ha producido en Estados Unidos, país que tiene un Sistema Sanitario radicalmente diferente al nuestro.

- No hay ningún estudio, en España ni en Europa, que compare de manera prospectiva y randomizada las diferentes técnicas y mucho menos que haga un análisis serio de costes.

- Aunque tuviéramos un análisis de costes detallado, es difícil con tan poco seguimiento sacar conclusiones veraces sobre la efectividad de la técnica.

- La mayoría de los centros (fuera de Estados Unidos) se encuentran aún en su curva de aprendizaje, con lo cual, los costes no son valorables, ya que es previsible que disminuyan sensiblemente.

Basándonos en estas premisas, hemos revisado algunos trabajos publicados en la literatura, no sólo en nuestra especialidad sino también en otras (cirugía cardíaca, cirugía general, etc.) para intentar inferir algo de luz sobre este, nada fácil, asunto. 


\section{ANÁLISIS DE COSTES}

La evaluación económica es el conjunto de procedimientos o técnicas de análisis dirigidos a evaluar el impacto de distintas opciones sobre el bienestar de la sociedad. Su objetivo último es ayudar a elegir racionalmente la mejor opción disponible, es decir, aquella que tenga el mayor impacto sobre el bienestar o sobre la salud. La economía de la salud no trata sólo de costes, sino también y de forma más importante, de beneficios ${ }^{15}$.

La evaluación económica se centra en la identificación, medida y valoración de los efectos que se supone tienen una relación directa con el bienestar, constituyendo un instrumento indispensable para mejorar la práctica sanitaria y para conseguir mejores resultados a un coste asumible por las comunidades.

Los efectos de la evaluación económica son de dos tipos: los efectos sobre los recursos o costes y los efectos sobre la salud o beneficios. Muchas veces se entienden como beneficios los efectos deseados o que tienen una asociación positiva con el bienestar (años y calidad de vida ganados, recursos ahorrados), y como costes los efectos no deseados o que tienen una asociación negativa con el bienestar (valor de los recursos empleados o empeoramiento de la salud) ${ }^{15}$.

Los costes se clasifican en directos, indirectos e intangibles. Los costes directos incluyen los gastos médicos y no médicos que forman parte integrante del proceso clínico que se evalúa. Los costes indirectos no se asocian al proceso evaluado sino a uno de sus efectos, como la pérdida de la capacidad productiva; suelen ser difíciles de medir y se incluyen según criterio personal ${ }^{16}$. Los costes intangibles se asocian a entidades inmateriales dificiles de cuantificar, como el dolor o la salud, y su inclusión en los análisis fármaco-económicos es excepcional, aunque deben tenerse en cuenta cuando se analiza la calidad de vida. Los costes directos son los que se suelen considerar más frecuentemente en los estudios fármaco-económicos ${ }^{17}$.

Los componentes relacionados directamente con el coste directo de un procedimiento quirúrgico concreto deben incluir los siguientes:

- Tiempo de utilización de quirófano

- Instrumental quirúrgico (fungible y amortización del reutilizable)
- Estancia hospitalaria

- Medicación (oral e intravenosa)

- Transfusión sanguínea

- Costes de los profesionales sanitarios

En el caso de la cirugía robótica, debemos añadir la compra del aparato así como el mantenimiento anual.

En un análisis realizado en dos grandes instituciones norteamericanas en el año 2004, la PRA ahorraba sobre la PRL 487\$ y sobre la PRR $1,726 \$$. Si excluían el coste de la compra del robot, esta diferencia entre la PRA y la PRR, se cifraba en $1.155 \$$. Los factores con más peso para explicar la diferencia fueron la amortización por paciente del coste del aparato así como el instrumental fungible. A pesar de que el tiempo de quirófano (140 vs. 160 min.) es favorable a la PRR y también la estancia hospitalaria ( 1.3 vs. 2.5 ), esto no era suficiente para compensar la diferencia ${ }^{18}$.

Para que los costes entre la PRA y la PRR se hubieran igualado, la estancia de la primera debería ser mayor de 5 días (6,3 exactamente) manteniéndose en 1.3 el de la PRR. Revisando las estancias medias de las series españolas publicadas (abiertas y laparoscópicas) vemos que en la mayoría de ellas la estancia es muy superior a los 5 dias ${ }^{19-22}$. Para hacerse una idea del significado económico que pudiera tener la reducción en la estancia en nuestro medio, Herranz et al. estimaron el coste de un día de ingreso hospitalario en un Servicio de Urología (Año 2003) en 298 euros $^{23}$. Por tanto, si las cifras de estancia disminuyeran de las publicadas (Rango 5-10) se podría ahorrar entre 1.192 y 2.682 euros respectivamente, por paciente e intervención.

Lotan et al. comunican que en su centro, en el momento de la publicación (2004), la única circunstancia que podría hacer equivalente, en términos de gasto, las tres técnicas, sería una reducción en el precio de equipo (robot) así como una disminución del coste del instrumental por cada caso $^{18}$. En nuestro medio, según datos recientes facilitados por Palex Medical S.A. (distribuidora del Sistema da Vinci en España), el coste del instrumental robótico y fungible por caso se calcula en aproximadamente 1.500-2.000 euros (mas IVA).

La Clínica Mayo estima que para hacer equivalentes en coste la PRA y la PRR en sus centros, 
el coste del material por paciente debería ser de $500 \$$, el precio del Robot de 500.000\$ y el mantenimiento anual de $34.000 \$^{18}$. En estos momentos, en nuestro país, estos costes son de aproximadamente 1,7 millones de euros (compra del Robot) y 125.000 euros (mantenimiento anual).

Con respecto al tiempo quirúrgico, los análisis de costes son difíciles de realizar. Si comparamos con las cifras de la literatura, muchas de ellas se refieren a series de uno o dos cirujanos expertos ${ }^{24-}$ 28 . En la práctica diaria existe una gran variabilidad basada en que en la mayoría de los Servicios los cirujanos (incluidos Médicos Residentes) no se dedican específicamente a una sola técnica y el número de procedimientos al año, no permite una real maestría. Además, se debe considerar la curva de aprendizaje de cada cirujano.

Está ampliamente demostrado que el tiempo quirúrgico al inicio de la curva de aprendizaje impacta de manera clara en los costes y que va disminuyendo con la experiencia. Burgess et al. han comunicado recientemente su análisis de costes entre PRA, PRR y PRP, comparando la curva de aprendizaje (20 primeros casos) con los siguientes, hasta un total de 110. Demuestran como, una vez adquirida una experiencia suficiente en el procedimiento, se puede reducir el coste hasta en un $27 \%^{29}$.

La duración de la intervención ha sido uno de los grandes caballos de batalla del abordaje mínimamente invasivo. Los tiempos iniciales de las series laparoscópicas eran tremendamente largos $^{12,30-32}$ aunque fueron mejorando a medida que avanzaba la curva de aprendizaje ${ }^{5,33}$. Con la cirugía robótica está sucediendo algo parecido, y los trabajos demuestran que el tiempo quirúrgico se acorta de manera significativa y exponencial cuando aumenta el número de $\operatorname{casos}^{29}$. Pero parece que las series iniciales de PRR comunican tiempos menores en muchos casos a los comunicados por cirujanos laparoscopistas expertos finalizada la curva de aprendizaje y muy similares a los tiempo medios de prostatectomía abierta de series recientes ${ }^{25}$.

Revisando algunas series laparoscópicas españolas recientes podemos ver cómo los tiempos quirúrgicos son una importante fuente de gasto. Se sitúan en torno a los 200 minutos y en más de 300 en los primeros $\operatorname{casos}^{20,21,34}$. Si mira- mos el coste de la hora de quirófano, Herranz et al. lo cifran en su centro en 2003 en aproximadamente 169 Euros, por tanto una reducción en el tiempo quirúrgico, supondría un ahorro importante ${ }^{23}$.

Parece que esta reducción en el tiempo quirúrgico a medida que se adquiere más experiencia también se demuestra en otros procedimientos robóticos no urológicos. Morgan et al. analizan su experiencia en dos procedimientos de cirugía cardíaca (reparación de la válvula mitral y cierre de defecto septal) con y sin robot. Sus conclusiones son que los costes hospitalarios (excluyendo la posible amortización por la adquisición del aparato) no aumentan con la cirugía robótica. Además, los costes se minimizan con el paso del tiempo, debido a la reducción del tiempo operatorio y del número de instrumentos necesarios para realizar la intervención ${ }^{35}$. Afirman, que para ellos, el procedimiento es coste-efectivo, ya que la calidad de vida de los pacientes tras la intervención es significativamente mejor ${ }^{36}$.

Ya se ha comentado que la tecnología robótica en urología también está teniendo una expansión hacia procedimientos no oncológicos, como la pieloplastia desmembrada. Existe mucha evidencia a cerca de la menor morbilidad del abordaje mínimamente invasivo en este tipo de procedimiento. Gettmann et al. demuestran que, en su experiencia, la pieloplastia da Vinci es 1,7 veces más rápida que la realizada por vía laparoscópica convencional. Esta reducción en el tiempo radica en la mayor sencillez para la sutura que el sistema da Vinci proporciona ${ }^{37}$.

\section{DISCUSION}

La implantación definitiva de una técnica o tecnología quirúrgica nueva, viene determinada, por dos hechos; el primero, que demuestre sus buenos resultados, a medio y largo plazo, tanto terapéutico como subjetivo para el paciente y el segundo su viabilidad económica en cuanto a sus costes.

Es de todo conocido, que cualquier tecnología nueva lleva detrás unos costes de investigación, desarrollo, marketing y comercialización, que acaban siendo imputados al consumidor. Estos costes, se minimizan con el tiempo y sobre todo con la aparición de competencia. 
Con el Sistema da Vinci, nos encontramos en la peor situación posible desde el punto de vista del coste-eficiencia, por una serie de razones:

- Es una tecnología muy novedosa

- Incorpora ventajas ciertamente innovadoras e interesantes

- Permite realizar procedimientos de gran dificultad técnica manteniendo un abordaje mínimamente invasivo

- Todos los datos publicados hasta la fecha apuntan a unos resultados comparables a los de las técnicas convencionales

- Presenta una gran demanda y aceptación social.

- Sólo existe una compañía en el mercado, es decir, domina el monopolio

Ante esto, es difícil demostrar ventajas económicas sobre técnicas absolutamente desarrolladas. Claramente la PRR, hoy en día, es más cara que las otras opciones quirúrgicas (PRA, PRL o PRP) como tratamiento del cáncer de próstata localizado.

No es nuestro objetivo hacer apología ciega de esta técnica pero sí lejos de una visión puramente economicista, valorar algunos hechos, que como médicos al cuidado de nuestros enfermos, debemos tener en cuenta.

- El gran caballo de batalla, para que los costes de este procedimiento se puedan equiparar a los que actualmente se están desarrollando en nuestros hospitales, es la compra del robot y su mantenimiento anual. Cuando alguno de nuestros hospitales compra un aparato de TC o de RMN nadie se plantea realizar una amortización económica en cada paciente de ese desembolso. Tan solo se piensa en dar una mejor calidad asistencial, basada en una mayor precisión diagnostica (menor radiación, mayor rapidez, etc.).

- Esta claro que en la Robótica estamos al principio de probablemente una larga historia. La mayoría del incremento de los costes (exceptuando el coste del Robot) se basa en el mayor tiempo operatorio (en relación a la PRA) que va disminuyendo a medida que se adquiere más experiencia $^{29,35}$ y en los costes de los materiales fungibles. Solamente disminuyendo la estancia se podría recuperar gran parte del dinero imputable a este costes.
- En la mayoría de los Centros el uso del robot es de carácter multidisciplinar. Esto puede ayudar al principio a cubrir los gastos de mantenimiento y alcanzar un mínimo número de casos anual (alrededor de 300).

- No debemos olvidar que la robótica está permitiendo tanto en Estados Unidos como en Europa, que muchos Urólogos (sin formación laparoscópica avanzada) ofrezcan a sus pacientes con cáncer de próstata un tratamiento mínimamente invasivo, con una más que razonable seguridad en los resultados.

- La robótica será imparable en la PR si se demuestra, cosa probable en un medio plazo, que logra mejores resultados funcionales (erección y continencia) que las técnicas clásicas abiertas o que la laparoscopia convencional. Lo que sí está fuera de duda es que consigue, al menos, resultados oncológicos equiparables a los de la PRA y PRL.

- Independientemente de la PRR, existen otras técnicas en nuestra especialidad (cistectomía radical, linfadenectomía pélvica, linfadenectomía retroperitoneal, cirugía renal compleja, etc.) en las cuales, la introducción de la cirugía laparoscópica está suponiendo un gran esfuerzo, debido, en gran medida, a que suponen un importante reto técnico. Con esta nueva tecnologia, se abre un horizonte alentador, aún por explorar, respecto a las ventajas que el robot nos puede aportar.

\section{CONCLUSIONES}

Un buen punto de partida, seria evaluar esta técnica en centros sanitarios terciarios (con experiencia amplia tanto en cirugía laparoscópica como abierta) de nuestra red sanitaria, de manera prospectiva y con instrumentos veraces y bien diseñados para medir tanto los costes como los resultados.

En cualquier caso, mas allá de las razones económicas de la implantación de esta alternativa, y dejando a un lado el torbellino mediático que se genera alrededor de cualquier tecnología nueva y llamativa, la comunidad urológica tiene la responsabilidad de intentar demostrar si todos estos avances que la técnica pone a disposición del cirujano pueden ser de utilidad para mejorar la calidad de vida de nuestros pacientes intervenidos de PR, mejorando sus resultados funcionales 
y logrando además reducir la recuperación postoperatoria y el dolor. ¿Puede y debe nuestro sistema de salud permitirse esto? Creemos que en un futuro, tal como avanza la técnica, la respuesta será si, porque entre otras cosas, lo demandará el usuario.

\section{REFERENCIAS}

1. Satava RM. Robotic surgery: from past to future-a personal journey. Surg Clin North Am. 2003 Dec;83(6):14911500, xii.

2. Gardner TA, Bissonette EA, Petroni GR, McClain R, Sokoloff MH, Theodorescu D. Surgical and postoperative factors affecting length of hospital stay after radical prostatectomy. Cancer. 2000 Jul 15;89(2):424-430.

3. Farnham SB, Webster TM, Herrell SD, Smith JA. Intraoperative blood loss and transfusion requirements for robotic-assisted radical prostatectomy versus radical retropubic prostatectomy. Urology. 2006 Feb;67(2):360363.

4. Menon M, Tewari A, Baize B, Guillonneau B, Vallancien G. Prospective comparison of radical retropubic prostatectomy and robot-assisted anatomic prostatectomy: the Vattikuti Urology Institute experience. Urology. 2002;60 (5): 864-868.

5. Salomon L, Sèbe P, De La Taille A, Vordos D, Hoznek A, Yiou $\mathrm{R}$ et al. Open versus laparoscopic radical prostatectomy: Part II. BJU Int. 2004;94(2): p. 244-250.

6. Smith JA. Robotically assisted laparoscopic prostatectomy: an assessment of its contemporary role in the surgical management of localized prostate cancer. Am J Surg. 2004 Oct; 188(4A Suppl):63S-67S.

7. Lotan Y, Gettman MT, Roehrborn CG, Pearle MS, Cadeddu JA. Cost comparison for laparoscopic nephrectomy and open nephrectomy: analysis of individual parameters. Urology. 2002 Jun;59(6):821-825.

8. Velidedeoglu E, Williams N, Brayman KL, Desai NM, Campos L, Palanjian M et al. Comparison of open, laparoscopic, and hand-assisted approaches to live-donor nephrectomy. Transplantation 2002 Jul 27;74(2):169-172.

9. Valdivia J.Rentabilidad economica de la C.M.I. Tema Monografico, in LIX Congreso Nacional de Urologia. 1994.

10. Rassweiler J, Hruza M, Teber D, Su LM. Laparoscopic and robotic assisted radical prostatectomy - critical analysis of the results. Eur Urol. 2006;49(4): p. 612-624.

11. Kawakita M, Sato M, Oguchi N, Muguruma K, Murota T, Matsuda T. Transperitoneal laparoscopic radical prostatectomy with the Montsouris technique: experience in initial 5 cases. Nippon Hinyokika Gakkai Zasshi. 2001 May;92(4):506-512.

12. Habuchi $T$, Iinuma $M$, Sato $K$, Sato $S$, Matsuura $S$, Shimoda N, et al. Early results in the initial 15 cases of laparoscopic radical prostatectomy in Akita University Medical Center. Hinyokika Kiyo. 2002,48(3): p.139-144.

13. Guillonneau B, Cathelineau X, Barret E, Rozet F, Vallancien G. Laparoscopic radical prostatectomy. Preliminary evaluation after 28 interventions. Presse Med, 1998; 27(31): p. 1570-1574.

14. Antiphon P, Hoznek A, Benyoussef A, de lataille A, Cicco A, Elard S et al. Complete solo laparoscopic radical prostatectomy: initial experience. Urology, 2003;61(4):724-728; discussion 728-729.
15. Abad Santos F, Fernández J, Martinez E, Galicia I, Farmacoeconomia: evaluacion economica de los medicamentos. Farmacoterapia. 1997;14:86-98.

16. Sacristan J, Badía X, Rovira J. Farmaco-economía: evaluación economica de medicamentos. ed. E. Medicos. 1995.

17. Rose DN. AIDS drug regimens that are worth their costs. JAMA. 1998 Jan 14;279(2):160-161.

18. Lotan Y, Cadeddu JA, Gettman MT. The new economics of radical prostatectomy: cost comparison of open, laparoscopic and robot assisted techniques. J Urol. 2004 Oct;172(4 Pt 1):1431-1435.

19. Aguiló Lucia F, Suárez Novo JF, Planes Morín J, Condom Mundó E. Prostatectomía radical. Revisión de nuestra serie en el periodo 1997-2003. Actas Urol Esp. 2005;29(6): 542-549.

20. Estébanez Zarranz J, Amón Sesmero J, Conde Redondo C, Santos Largo J, Alonso Fernández D, Robles Samaniego A, et al. Prostatectomía radical laparoscópica: experiencia preliminar. Actas Urol Esp. 2003 May;27(5):370-378.

21. Pascual Piédrola JI, Grasa Lanau V, Cuesta Alcalá JA, Aldave Villanueva J, Ponz González M, Ipiens Aznar A. Prostatectomía radical laparoscópica. ¿Sólo en Centros de excelencia?. Actas Urol Esp. 2005 Nov-Dec;29(10):927933; discussion 933.

22. Portillo Martín JA, Rado Velázquez M, Gutiérrez Baños JL, Martín García B, Hernández Rodríguez R, del Valle Schaan JI, et al. Complicaciones de una serie consecutiva de 133 casos de prostatectomía radical. Actas Urol Esp. 2001 Sep;25(8):559-566.

23. Herranz Amo F, Subirá Ríos D, Hernández Fernández C, Martínez Salamanca JI, Monzó JI, Cabello Benavente R. Comparacion de costes entre la nefrectomia radical laparoscopica y la abierta en el adenocarcinoma renal. Actas Urol Esp. 2006 Oct;30(9):921-925.

24. Salomon L, Sèbe P, De la Taille A, Vordos D, Hoznek A, Yiou R, et al. Open versus laparoscopic radical prostatectomy: part I. BJU Int. 2004 Jul;94(2):238-243.

25. Ficarra V, Cavalleri S, Novara G, Aragona M, Artibani W. Evidence from robot-assisted laparoscopic radical prostatectomy: a systematic review. Eur Urol. 2007 Jan;51(1):4555; discussion 56. Epub 2006 Jun 30.

26. Catalona WJ, Carvalhal GF, Mager DE, Smith DS. Potency, continence and complication rates in 1,870 consecutive radical retropubic prostatectomies. J Urol. 1999 Aug; 162(2):433-438.

27. Guillonneau B, el-Fettouh H, Baumert H, Cathelineau X, Doublet JD, Fromont G et al. Laparoscopic radical prostatectomy: oncological evaluation after 1,000 cases a Montsouris Institute. J Urol. 2003 Apr;169(4):1261-1266.

28. Menon M, Shrivastava A, Kaul S, Badani KK, Fumo M, Bhandari $\mathrm{M}$, et al. Vattikuti Institute Prostatectomy: Contemporary Technique and Analysis of Results. Eur Urol. 2007 Mar;51(3):648-57; discussion 657-658. Epub 2006 Nov 3.

29. Burgess SV, Atug F, Castle EP, Davis R, Thomas R. Cost analysis of radical retropubic, perineal, and robotic prostatectomy. J Endourol. 2006 Oct;20(10):827-830.

30. Kawabata G, Hara I, Hara S, Isotani S, Sakai Y, Wada Y, et al. Laparoscopic radical prostatectomy: initial 17 case report. Nippon Hinyokika Gakkai Zasshi. 2001 Nov;92(7): 647-655.

31. Jacob F, Salomon L, Hoznek A, Bellot J, Antiphon P, Chopin DK, Abbou CC. Laparoscopic radical prostatectomy: preliminary results. Eur Urol. 2000 May;37(5):615-620. 
32. Hattori R, Ono Y, Gotho M, Yoshikawa Y, Hirabayashi S, Yamada S, Ohshima S. Laparoscopic radical prostatectomy-initial 10 cases. Nippon Hinyokika Gakkai Zasshi. 2001 Sep;92(6):603-608.

33. Türk I, Deger S, Winkelmann B, Schönberger B, Loening SA. Laparoscopic radical prostatectomy. Technical aspects and experience with 125 cases. Eur Urol. 2001 Jul;40(1): 46-52; discussion 53.

34. Cansino Alcaide JR, Alvarez Maestro M, Castillo Cabrera PM, Martínez-Piñeiro Lorenzo L, Tabernero Prieto A, De la Peña Barthel JJ. Prostatectomía radical laparoscópica. Revisión de la literatura. Nuestra experiencia. Actas Urol Esp. 2006 May;30(5):517-530.

35. Morgan JA, Thornton BA, Peacock JC, Hollingsworth KW, Smith CR, Oz MC, Argenziano M. Does robotic technology make minimally invasive cardiac surgery too expensive? A hospital cost analysis of robotic and conventional techniques. J Card Surg. 2005 May-Jun;20(3):246-251.
36. Horgan JA, Hollingsworth KW, Colleti NJ et al. Postoperative pain and quality of life in patients undergoing atrial septal repair using minimally invasive and robotic techniques, in Heart Surgery Forum. 2003.

37. Gettman MT, Neururer R, Bartsch G, Peschel R. AndersonHynes dismembered pyeloplasty performed using the da Vinci robotic system. Urology. 2002 Sep;60(3):509-513.

Correspondencia autor: Dr. J.I. Martínez-Salamanca Department of Urology.

Weill Medical College at Cornell University. New York E-mail autor: msalamanca99@hotmail.com Información artículo: Original 